\title{
Molecular-Field-Based Three-Dimensional Similarity Studies on Quinoline-Based CNS Active Agents
}

\begin{abstract}
Alka Bali
University Institute of Pharmaceutical Sciences, Panjab University, Chandigarh 160014, India

Correspondence should be addressed to Alka Bali, alka.bali@rediffmail.com

Received 9 June 2011; Accepted 6 July 2011

Academic Editors: B. Nigovic, R. Veerasamy, and C. Zacharis

Copyright () 2011 Alka Bali. This is an open access article distributed under the Creative Commons Attribution License, which permits unrestricted use, distribution, and reproduction in any medium, provided the original work is properly cited.

A series of quinoline-based agents with CNS activity have been analyzed for their three-dimensional similarity with respect to a set of standard atypical antipsychotics. The method aligns the molecules based on their molecular fields represented as local extrema of electrostatic, van der Waals, and hydrophobic potentials of the molecule termed "field points." The compounds in the series were found to demonstrate relatively lesser 3D similarity to the dibenzodiazepine derivative clozapine. Similarity values were higher with respect to extended chain compounds ketanserin, ziprasidone, and risperidone. The results obtained were found to agree with the physicochemical similarity of the compounds reported earlier.
\end{abstract}

\section{Introduction}

Molecular similarity is one of the most widely used concepts in the computer-aided approaches to molecular design. According to the "molecular similarity principle," compounds with similar chemical structures are more likely to possess similar physicochemical and biological activities [1-6]. Despite recent examples that one cannot conclude property similarity from structural similarity in every case [7], this is still the underlying assumption of current drug design efforts which includes building QSAR models and using them for making predictions for new compounds. Some studies also indicate that structural similarity does not always imply similarity in descriptors [8].

The concept of chemical similarity does not have a formal definition, and it may vary depending upon the application for which similarity needs to be determined. Quantification of chemical similarity is based on numerical representation of a chemical structure, and similarity comparisons are done based on 2D or 3D approaches. 2D approaches involve the calculation of fingerprint-based [9] or descriptor-based similarity [2]. The former involves a simple count of shared features (common fragment substructures) as a measure of quantifying the degree of structural resemblance. In the latter case, similarity can be based on descriptors such as continuous whole molecule properties, for example, $\log P$, molar refractivity, and topological indexes. Various similarity metrics exist that return a score indicating the level of similarity between molecules under comparison. Frequently used metrics are simple distance measures such as Hamming and Euclidean distance and association coefficients such as Tanimoto, Dice, and Cosine coefficients [10]. Tanimoto coefficient has been the measure of choice for fragmentbased chemical similarity work, whereas euclidean distance measurement is the most popular measure for continuous data (descriptors).

3D methods can be alignment-independent methods based on descriptors such as geometric atom pairs and their distances, valence and torsion angles, and atom triplets and alignment methods, which consider the conformational flexibility of the molecules. Field-based alignment methods [11] are based on quantum mechanical calculations, and the similarity score is related to the electron density of the molecules which involves calculation of steric fields (van der Waals surface) and electrostatic fields (derived from precalculated point charges). Examples include methods like MIMIC [12] using fixed conformations in matching process. Flexiblefield-based alignment (FLASHFLOOD) [13] is preferred over conformationally rigid matching $[14,15]$. Comparable activities of diverse molecules at the same molecular targets can be explained by considering the molecules' fields rather than their atomic structure because the field pattern is a far 


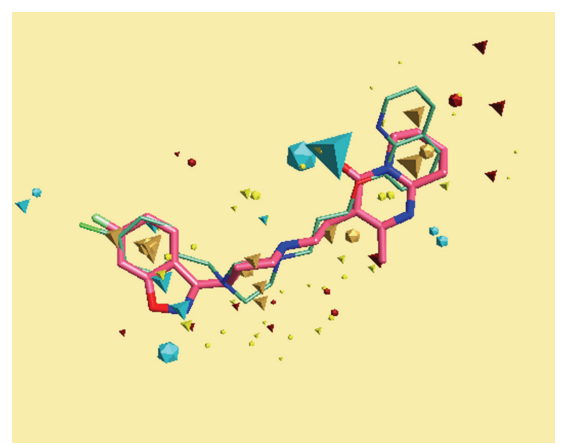

(a)

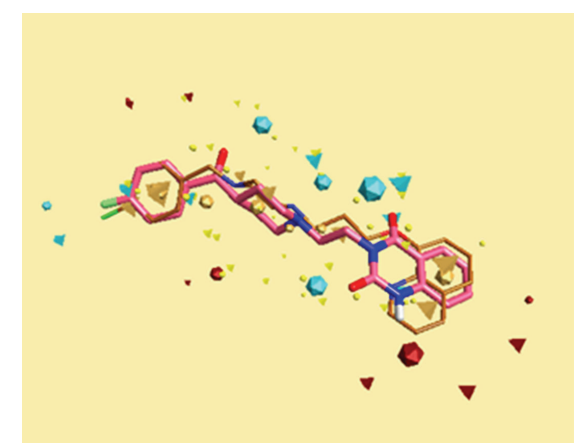

(b)

FIGURE 1: Alignment of compound $\mathbf{1 4}$ (thin sticks) with risperidone (a) and ketanserin (b). Risperidone and ketanserin are shown as capped sticks (pink). Tetrahedra and dodecahedra depict field points for 14 and reference compounds, respectively. Blue, maroon, yellow, and brown colors depict negative field, positive field, surface field, and hydrophobic field points.

TABLE 1: Chemical structures and results in pharmacological testing [16].

\begin{tabular}{llllc}
\hline Compound no. & $\mathrm{X}$ & $\mathrm{Y}$ & $\mathrm{Ar}$ & $\begin{array}{c}\text { Atypical antipsychotic } \\
\text { profile }\end{array}$ \\
\hline $\mathbf{7}$ & $\mathrm{H}$ & $\mathrm{C}=\mathrm{O}$ & 6-quinolyl & $-{ }^{*}$ \\
$\mathbf{8}$ & $\mathrm{H}$ & $\mathrm{CH}_{2}$ & 6-quinolyl & - \\
$\mathbf{9}$ & $\mathrm{H}$ & $\mathrm{C}=\mathrm{O}$ & 8-quinolyl & $-{ }^{*}$ \\
$\mathbf{1 0}$ & $\mathrm{H}$ & $\mathrm{CH}$ & 8-quinolyl & + \\
$\mathbf{1 1}$ & $\mathrm{Cl}$ & $\mathrm{C}=\mathrm{O}$ & 6-quinolyl & $-{ }^{*}$ \\
$\mathbf{1 2}$ & $\mathrm{Cl}$ & $\mathrm{CH} 2$ & 6-quinolyl & - \\
$\mathbf{1 3}$ & $\mathrm{Cl}$ & $\mathrm{C}=\mathrm{O}$ & 8-quinolyl & $-{ }^{*}$ \\
$\mathbf{1 4}$ & $\mathrm{Cl}$ & $\mathrm{CH}$ & 8-quinolyl & + \\
\hline
\end{tabular}

* Show blockade only in apomorphine-induced stereotypy assay indicative of potential to cause extrapyramidal symptoms.

superior description of molecule's binding properties than its atomic structure. Compounds which are structurally diverse but show comparable activity have similar fields and, hence, similar binding properties so that these can bind to the same target site and elicit the same biological effect.

We had recently reported a novel series of quinoline derivatives evaluated for their atypical antipsychotic potential [16] and further assessed for their 2D similarity (physicochemical similarity) with respect to the standard atypical antipsychotic drugs. In the present research paper, we report the 3D similarity (based on field similarity, volume similarity, and shape similarity) of this series of compounds to the standard drugs clozapine, risperidone, ziprasidone, and ketanserin based upon field-based alignment methods.

\section{Method}

Field based similarity of the compound set was assessed with respect to the standard drugs taken as reference, that is, clozapine, ketanserin, ziprasidone and risperidone using
FieldAlign2.1.1 (Cresset BioMolecular Discovery Ltd., UK). The three dimensional models of the reference drugs were generated using ChemBio3D Ultra 12.0 and energy minimization was performed with MM2 force field to minimum RMS gradient of 0.100 . These reference drugs in a defined $3 \mathrm{D}$ conformation were then imported in sdf (MDL mol) format to FieldAlign2.1.1. Molecules to be aligned were imported in 2D from ChemBioDraw Ultra 12.0 as sdf (MDL mol) files. The maximum number of conformations generated for any molecule was limited to 200 in order to have a balance of the quality of alignments and calculation time. Number of high temperature dynamics for flexible rings was set at 5. Gradient cut-off for conformer minimization was 0.5. Coarseness of the sampling of conformational space was controlled by filtering duplicate conformers at rms 0.5. Standard scoring function was used based on 50\% shape similarity and 50\% dice volume similarity to derive overall similarity between two conformations. Further, a 3D reference template was generated taking three reference drug molecules at a time (loaded as single 2D structures ) using FieldTemplator2.1.1 (Cresset BioMolecular Discovery Ltd., UK) that searches for common field patterns across the explored conformational space of a set of ligands looking for commonality. The best template was selected based on their field similarity, shape similarity and overall similarity scores.

\section{Results and Discussion}

Table 1 shows the chemical structures and pharmacological activity profile of the quinoline-based compound set (7-14) taken for the 3D alignment studies. The overall alignment (similarity) scores along with the corresponding field similarity, volume similarity, and shape similarity scores of the test molecules with respect to a selection of standard drugs are shown in Tables 2, 3, and 4. The alignment scores serve as a measure of how similar the molecular fields of the two molecules are in the given alignment.

Figures 1-3 show the graphic display of the best alignment (highest alignment score) of the compound 14 with standard drugs along with the various field points. Compound $\mathbf{1 4}$ is the lead compound which had demonstrated 
TABLE 2: Similarity of test compounds with respect to risperidone and ketanserin.

\begin{tabular}{|c|c|c|c|c|c|c|c|c|c|c|c|c|c|c|}
\hline \multirow[t]{2}{*}{ Compd. no. } & \multicolumn{2}{|c|}{ Similarity } & \multicolumn{2}{|c|}{ Field similarity } & \multicolumn{2}{|c|}{ Field score } & \multicolumn{2}{|c|}{ Vol. Similarity } & \multicolumn{2}{|c|}{ Volume score } & \multicolumn{2}{|c|}{ Shape similarity } & \multicolumn{2}{|c|}{ Shape score } \\
\hline & Ris. & Ket. & Ris. & Ket. & Ris. & Ket. & Ris. & Ket. & Ris. & Ket. & Ris. & Ket. & Ris. & Ket. \\
\hline 7 & 0.620 & 0.672 & 0.483 & 0.539 & -48.519 & -52.436 & 0.806 & 0.787 & 204.854 & 193.544 & 0.756 & 0.804 & 192.146 & 197.828 \\
\hline 8 & 0.619 & 0.648 & 0.541 & 0.480 & -50.853 & -46.808 & 0.709 & 0.784 & 201.876 & 191.679 & 0.697 & 0.815 & 175.958 & 199.110 \\
\hline 9 & 0.659 & 0.686 & 0.552 & 0.576 & -55.523 & -57.483 & 0.783 & 0.802 & 198.906 & 197.155 & 0.766 & 0.795 & 193.085 & 193.834 \\
\hline 10 & 0.610 & 0.683 & 0.525 & 0.565 & -49.877 & -55.710 & 0.748 & 0.772 & 189.082 & 188.609 & 0.696 & 0.801 & 175.690 & 195.756 \\
\hline 11 & 0.661 & 0.689 & 0.542 & 0.594 & -53.226 & -59.520 & 0.740 & 0.799 & 194.220 & 203.325 & 0.781 & 0.783 & 205.048 & 199.232 \\
\hline 12 & 0.626 & 0.659 & 0.512 & 0.501 & -48.374 & -49.907 & 0.770 & 0.803 & 200.828 & 202.951 & 0.740 & 0.818 & 193.227 & 206.720 \\
\hline 13 & 0.676 & 0.721 & 0.581 & 0.635 & -56.250 & -64.061 & 0.824 & 0.820 & 216.246 & 208.667 & 0.772 & 0.808 & 202.659 & 205.442 \\
\hline 14 & 0.658 & 0.716 & 0.549 & 0.610 & -53.264 & -58.534 & 0.745 & 0.802 & 194.555 & 202.734 & 0.768 & 0.823 & 200.627 & 208.108 \\
\hline Average & 0.641 & 0.684 & 0.536 & 0.563 & -51.986 & -55.557 & 0.766 & 0.796 & 200.070 & 198.583 & 0.747 & 0.806 & 192.305 & 200.754 \\
\hline
\end{tabular}

TABLE 3: Similarity of test compounds with respect to ziprasidone and clozapine.

\begin{tabular}{|c|c|c|c|c|c|c|c|c|c|c|c|c|c|c|}
\hline \multirow[t]{2}{*}{ Compd. no. } & \multicolumn{2}{|c|}{ Similarity } & \multicolumn{2}{|c|}{ Field similarity } & \multicolumn{2}{|c|}{ Field score } & \multicolumn{2}{|c|}{ Vol. similarity } & \multicolumn{2}{|c|}{ Volume score } & \multicolumn{2}{|c|}{ Shape similarity } & \multicolumn{2}{|c|}{ Shape score } \\
\hline & Zip. & Clz. & Zip. & Clz. & Zip. & Clz. & Zip. & Clz. & Zip. & Clz. & Zip. & Clz. & Zip. & Clz. \\
\hline 7 & 0.696 & 0.566 & 0.639 & 0.540 & -66.121 & -50.871 & 0.798 & 0.620 & 201.876 & 141.337 & 0.752 & 0.592 & 190.325 & 134.964 \\
\hline 8 & 0.651 & 0.567 & 0.564 & 0.482 & -58.038 & -41.151 & 0.741 & 0.649 & 186.210 & 147.078 & 0.738 & 0.652 & 185.787 & 147.697 \\
\hline 9 & 0.652 & 0.558 & 0.557 & 0.521 & -58.139 & -47.685 & 0.772 & 0.589 & 195.325 & 134.285 & 0.747 & 0.595 & 187.604 & 134.470 \\
\hline 10 & 0.632 & 0.579 & 0.575 & 0.484 & -59.279 & -43.358 & 0.708 & 0.586 & 178.021 & 132.880 & 0.689 & 0.674 & 173.209 & 152.661 \\
\hline 11 & 0.703 & 0.583 & 0.603 & 0.553 & -59.824 & -49.015 & 0.730 & 0.624 & 190.885 & 147.619 & 0.802 & 0.622 & 209.751 & 147.293 \\
\hline 12 & 0.603 & 0.590 & 0.457 & 0.556 & -45.789 & -48.918 & 0.778 & 0.569 & 202.276 & 133.575 & 0.749 & 0.625 & 194.708 & 146.938 \\
\hline 13 & 0.675 & 0.571 & 0.599 & 0.556 & -62.140 & -52.714 & 0.786 & 0.615 & 205.549 & 145.517 & 0.711 & 0.585 & 185.959 & 138.252 \\
\hline 14 & 0.675 & 0.573 & 0.606 & 0.539 & -63.004 & -49.314 & 0.730 & 0.654 & 189.892 & 153.728 & 0.744 & 0.606 & 193.411 & 142.511 \\
\hline Average & 0.661 & 0.573 & 0.575 & 0.529 & -59.042 & -47.878 & 0.755 & 0.613 & 193.754 & 144.002 & 0.742 & 0.619 & 190.094 & 143.098 \\
\hline
\end{tabular}

a potential atypical antipsychotic profile in our earlier studies. The best alignment obtained with risperidone shows the field superposition of quinoline and piperazine nitrogens in 14 with corresponding nitrogens in the drug. The overlap of negative field points corresponding to the ether oxygen in 14 and carbonyl oxygen in the drug is also seen as a major contributor to field similarity. The quinoline nitrogen does not, however, assume correspondence with any of the two heterocyclic nitrogens in risperidone. However, good intersection of quinoline and quinazoline nitrogens is seen in addition to the superposition of negative field points of ether moiety and carbonyl group, piperazine and piperidine nitrogens, and the corresponding halogen atoms in 14 and ketanserin, respectively, which accounts for higher similarity of $\mathbf{1 4}$ to ketanserin than to risperidone.

The best alignment of 14 with ziprasidone shows the overlay of field points corresponding to ether oxygen in 14 with benzothiazole nitrogen in the drug. Further, field superposition of chlorine in $\mathbf{1 4}$ with carbonyl oxygen of indolinone moiety in the drug contributes towards the overall similarity between the two. In comparison to these drugs, there is less superposition of the field points of $\mathbf{1 4}$ with clozapine accounting for lower similarity scores in this case. Further, the alignment of 14 with the three compound template generated from risperidone, ketanserin and ziprasidone does not improve the similarity scores as evident from the graphic display in Figure 3.
The overall similarity scores of the compounds were found to increase with chlorination with respect to risperidone, ziprasidone and ketanserin. However, compounds 10 and $\mathbf{1 4}$ showed nearly same similarity values with respect to clozapine.

The 8-quinoline based compounds were found to have higher similarity score with respect to risperidone and ketanserin than the corresponding 6-quinoline derivatives. However, with respect to ziprasidone, 6-quinoline compounds have a higher similarity (except 14 versus 12). Further, a pattern of increase in similarity scores with respect to the selected standard drugs was noted which was different for the 8- and 6-quinoline series. The similarity for the former was in the order clozapine $<$ risperidone $<$ ziprasidone $<$ ketanserin. Order for the 6-quinoline series was clozapine $<$ risperidone $<$ ketanserin $<$ ziprasidone.

The field similarity with respect to ziprasidone increased with chlorination for 8-quinoline derivatives and decreased for 6-quinoline derivatives. With respect to the other drug molecules, chlorination in general, increased the field similarity of the compounds. A comparison of 6- and 8-quinoline derivatives in terms of their field similarity scores showed that 8-quinoline derivatives have a greater field similarity to ziprasidone than their corresponding 6-quinoline analogs. However, with respect to clozapine, scores were not significantly different.

Shape similarity with respect to risperidone and ziprasidone (except 8-quinolyl benzoyl derivative 7) showed an 


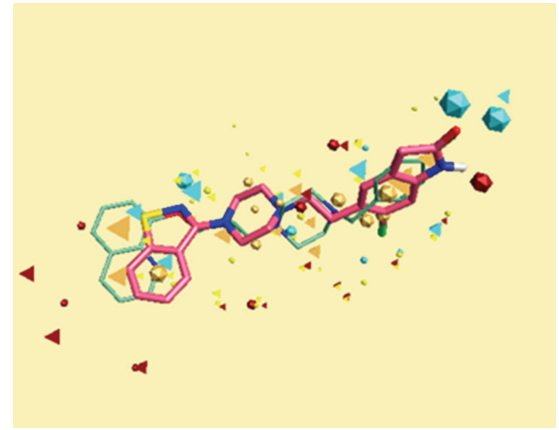

(c)

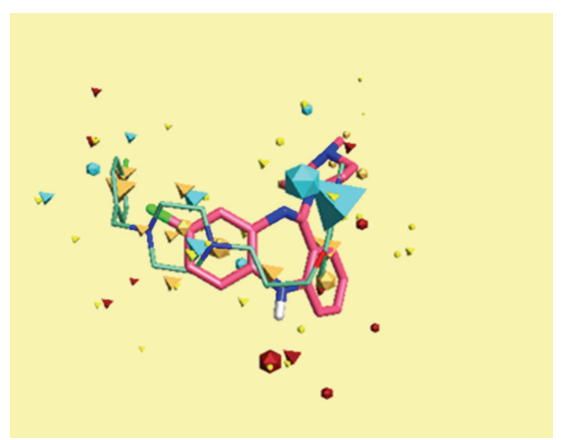

(d)

FIGURE 2: Alignment of compound 14 (thin sticks) with ziprasidone (c) and clozapine (d). Ziprasidone and clozapine are shown as capped sticks (pink). Field point depiction is the same as in Figure 1.

TABLE 4: Similarity of test compounds with respect to chlorpromazine and template.

\begin{tabular}{|c|c|c|c|c|c|c|c|c|c|c|c|c|c|c|}
\hline \multirow[t]{2}{*}{ Compd. no. } & \multicolumn{2}{|c|}{ Similarity } & \multicolumn{2}{|c|}{ Field similarity } & \multicolumn{2}{|c|}{ Field score } & \multicolumn{2}{|c|}{ Vol. similarity } & \multicolumn{2}{|c|}{ Volume score } & \multicolumn{2}{|c|}{ Shape similarity } & \multicolumn{2}{|c|}{ Shape score } \\
\hline & $\mathrm{CPZ}$ & Temp & $\mathrm{CPZ}$ & Temp & $\mathrm{CPZ}$ & Temp & $\mathrm{CPZ}$ & Temp & $\mathrm{CPZ}$ & Temp & $\mathrm{CPZ}$ & Temp & $\mathrm{CPZ}$ & Temp \\
\hline 7 & 0.613 & 0.603 & 0.535 & 0.466 & -48.258 & -47.987 & 0.628 & 0.620 & 141.826 & 141.337 & 0.690 & 0.740 & 164.092 & 185.972 \\
\hline 8 & 0.565 & 0.579 & 0.487 & 0.469 & -41.443 & -46.261 & 0.641 & 0.649 & 147.210 & 147 & 0.643 & 0.689 & 151.980 & 172.277 \\
\hline 9 & 0.557 & 0.575 & 0.532 & 0.451 & -49.327 & -45.631 & 0.572 & 0.589 & 135.325 & 134.285 & 0.583 & 0.699 & 137.385 & 174.194 \\
\hline 10 & 0.561 & 0.576 & 0.517 & 0.425 & -46.640 & -41.811 & 0.588 & 0.586 & 138.021 & 132.880 & 0.606 & 0.728 & 143.205 & 181.874 \\
\hline 11 & 0.543 & 0.596 & 0.493 & 0.456 & -46.066 & -44.811 & 0.630 & 0.624 & 141.855 & 147.619 & 0.592 & 0.736 & 145.862 & 191.305 \\
\hline 12 & 0.581 & 0.582 & 0.500 & 0.436 & -43.990 & -44.166 & 0.578 & 0.569 & 136.276 & 133.575 & 0.662 & 0.728 & 162.107 & 188.021 \\
\hline 13 & 0.599 & 0.635 & 0.520 & 0.543 & -48.090 & -54.255 & 0.616 & 0.615 & 145.549 & 145.517 & 0.679 & 0.726 & 167.193 & 188.809 \\
\hline 14 & 0.591 & 0.618 & 0.538 & 0.489 & -51.045 & -49.919 & 0.630 & 0.654 & 142.092 & 153.728 & 0.644 & 0.748 & 157.594 & 193.364 \\
\hline Average & 0.576 & 0.596 & 0.515 & 0.467 & -46.857 & -46.855 & 0.610 & 0.613 & 141.019 & 142.002 & 0.637 & 0.724 & 153.677 & 184.477 \\
\hline
\end{tabular}

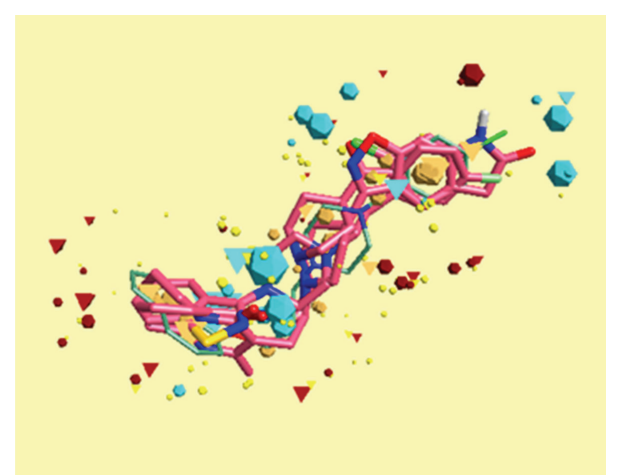

FIGURE 3: Alignment of compound 14 (thin sticks) with three compound templates generated from risperidone, ketanserin, and ziprasidone shown as capped sticks (pink). Field point depiction is the same as in Figure 1.

increase with chlorination. No particular pattern was seen on chlorination with respect to ketanserin. A comparison between 6- and 8-quinolyl derivatives showed that, for nonchlorinated compounds, the latter showed higher shape similarity to ketanserin and clozapine whereas, for chlorinated analogs, 6-quinoline derivatives showed higher scores.

Further, as expected, the volume similarity values were not significantly changed on chlorination or in the comparison of the 6-and 8-quinolyl derivatives.
The average similarity scores for the compounds were the highest with respect to ketanserin followed by ziprasidone and risperidone. Interestingly, the physicochemical similarity for the compounds reported earlier was the lowest with respect to ketanserin for the same drug group (90.64\%, $85.5 \%$, and $76.7 \%$ with respect to ziprasidone, risperidone and ketanserin, resp.). In line with the results from our previous computational studies, wherein the physicochemical similarity was the lowest with respect to the more compact dibenzodiazepine derivative clozapine (56.85\%), the 3D similarity values were also found to be the lowest with respect to clozapine as well as the conventional neuroleptic chlorpromazine.

An analysis of the breakup of the overall alignment scores shows that the contribution of the shape and volume similarity was significantly greater than the corresponding field similarity values in all the cases. As can be seen from the tables, the shape similarity and volume similarity is more than 0.7 for all the drug examples (except clozapine) whereas their field similarity scores range from 0.54 to 0.58 .

\section{Conclusions}

In the present work, a set of quinolyloxypropyl piperazine derivatives have been analyzed for their three-dimensional similarity to a selection of atypical antipsychotic drugs, and these demonstrate higher similarity with respect to the 
extended chain structures such as risperidone, ziprasidone, and ketanserin and lesser similarity to the prototypic agent clozapine. Further, specific patterns were observed for the change in similarity scores with change in chemical structure. An introspection of the field alignments obtained for the molecules (especially, the lead compound from our studies 14) with respect to standard drugs suggests a good correspondence of quinoline nitrogen, piperazine system, ether oxygen, and chlorine atom with the corresponding groupings in the standard drug molecules. This highlights the importance of these structural features as a part of the chromophoric system involved in the pharmacological activity of this class of compounds. Further development of this compound series can be carried out by appropriate modifications whilst retaining these salient features. Hence, the information generated from the molecular field analysis of this compound series can be used as a valuable tool for designing novel analogues by interpretation of their pharmacological activity in terms of their field pattern.

\section{Conflict of Interests}

The author declares no conflict of interests.

\section{References}

[1] N. Nikolova and J. Jaworska, "Approaches to measure chemical similarity: a review," QSAR and Combinatorial Science, vol. 22, no. 9-10, pp. 1006-1026, 2004.

[2] R. C. Glen and S. E. Adams, "Similarity metrics and descriptor spaces: which combinations to choose?" QSAR and Combinatorial Science, vol. 25, no. 12, pp. 1133-1142, 2006.

[3] A. Bender and R. C. Glen, "Molecular similarity: a key technique in molecular informatics," Organic and Biomolecular Chemistry, vol. 2, no. 22, pp. 3204-3218, 2004.

[4] D. E. Patterson, R. D. Cramer, A. M. Ferguson, R. D. Clark, and L. E. Weinberger, "Neighborhood behavior: a useful concept for validation of "molecular diversity" descriptors," Journal of Medicinal Chemistry, vol. 39, no. 16, pp. 3049-3059, 1996.

[5] P. Willett, J. M. Barnard, and G. M. Downs, "Chemical similarity searching," Journal of Chemical Information and Computer Sciences, vol. 38, no. 6, pp. 983-996, 1998.

[6] M. A. Johnson and G. M. Maggiora, Concepts and Applications of Molecular Similarity, John Wiley \& Sons, New York, NY, USA, 2005.

[7] Y. C. Martin, J. L. Kofron, and L. M. Traphagen, "Do structurally similar molecules have similar biological activity?" Journal of Medicinal Chemistry, vol. 45, no. 19, pp. 4350-4358, 2002.

[8] H. Kubinyi, "Similarity and dissimilarity: a medicinal chemist's view," Perspectives in Drug Discovery and Design, vol. 911, no. 0, pp. 225-252, 1998.

[9] D. R. Flower, "On the properties of bit string-based measures of chemical similarity," Journal of Chemical Information and Computer Sciences, vol. 38, no. 3, pp. 379-386, 1998.

[10] J. D. Holliday, C. Y. Hu, and P. Willett, "Grouping of coefficients for the calculation of inter-molecular similarity and dissimilarity using 2D fragment bit-strings," Combinatorial Chemistry and High Throughput Screening, vol. 5, no. 2, pp. 155-166, 2002.
[11] J. Mestres, D. C. Rohrer, and G. M. Maggiora, "A molecularfield-based similarity study of non-nucleoside HIV-1 reverse transcriptase inhibitors," Journal of Computer-Aided Molecular Design, vol. 13, no. 1, pp. 79-93, 1999.

[12] J. Mestres, D. C. Rohrer, and G. M. Maggiora, "MIMIC: a molecular-field matching program: exploiting applicability of molecular similarity approaches," Journal of Computational Chemistry, vol. 18, no. 7, pp. 934-954, 1997.

[13] M. C. Pitman, W. K. Huber, H. Horn, A. Krämer, J. E. Rice, and W. C. Swope, "Flashflood: a 3D field-based similarity search and alignment method for flexible molecules," Journal of Computer-Aided Molecular Design, vol. 15, no. 7, pp. 587612, 2001.

[14] J. Mestres, D. C. Rohrer, and G. M. Maggiora, "A molecularfield-based similarity study of non-nucleoside HIV-1 reverse transcriptase inhibitors: 2 . The relationship between alignment solutions obtained from conformationally rigid and flexible matching," Journal of Computer-Aided Molecular Design, vol. 14 , no. 1 , pp. 39-51, 2000.

[15] D. A. Thorner, D. J. Wild, P. Willett, and P. M. Wright, "Similarity searching in files of three-dimensional chemical structures: flexible field-based searching of molecular electrostatic potentials," Journal of Chemical Information and Computer Sciences, vol. 36, no. 4, pp. 900-908, 1996.

[16] A. Bali, S. Malhotra, H. Dhir, A. Kumar, and A. Sharma, "Synthesis and evaluation of 1-(quinoliloxypropyl)-4-aryl piperazines for atypical antipsychotic effect," Bioorganic and Medicinal Chemistry Letters, vol. 19, no. 11, pp. 3041-3044, 2009. 

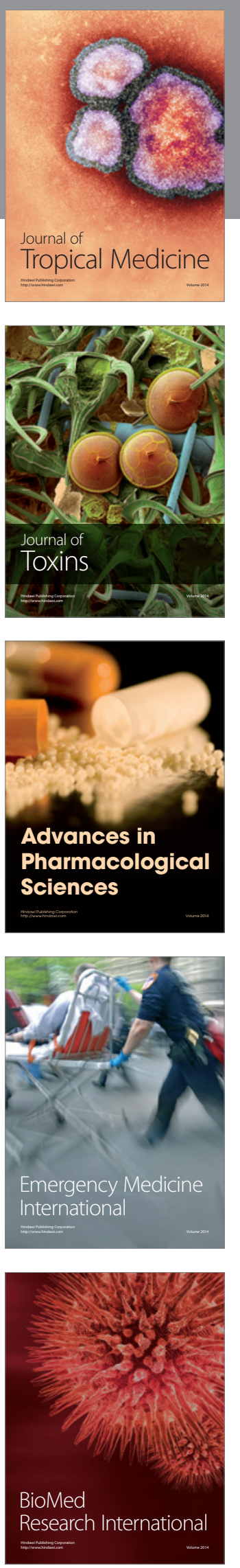
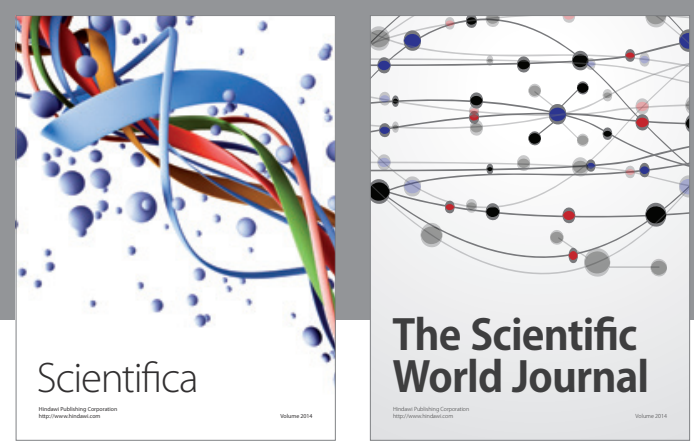

The Scientific World Journal
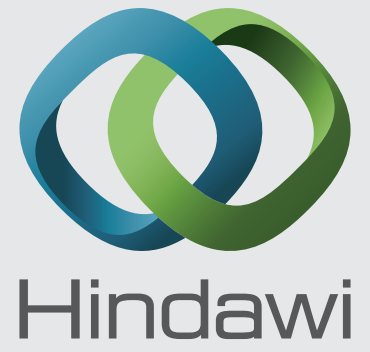

Submit your manuscripts at

http://www.hindawi.com
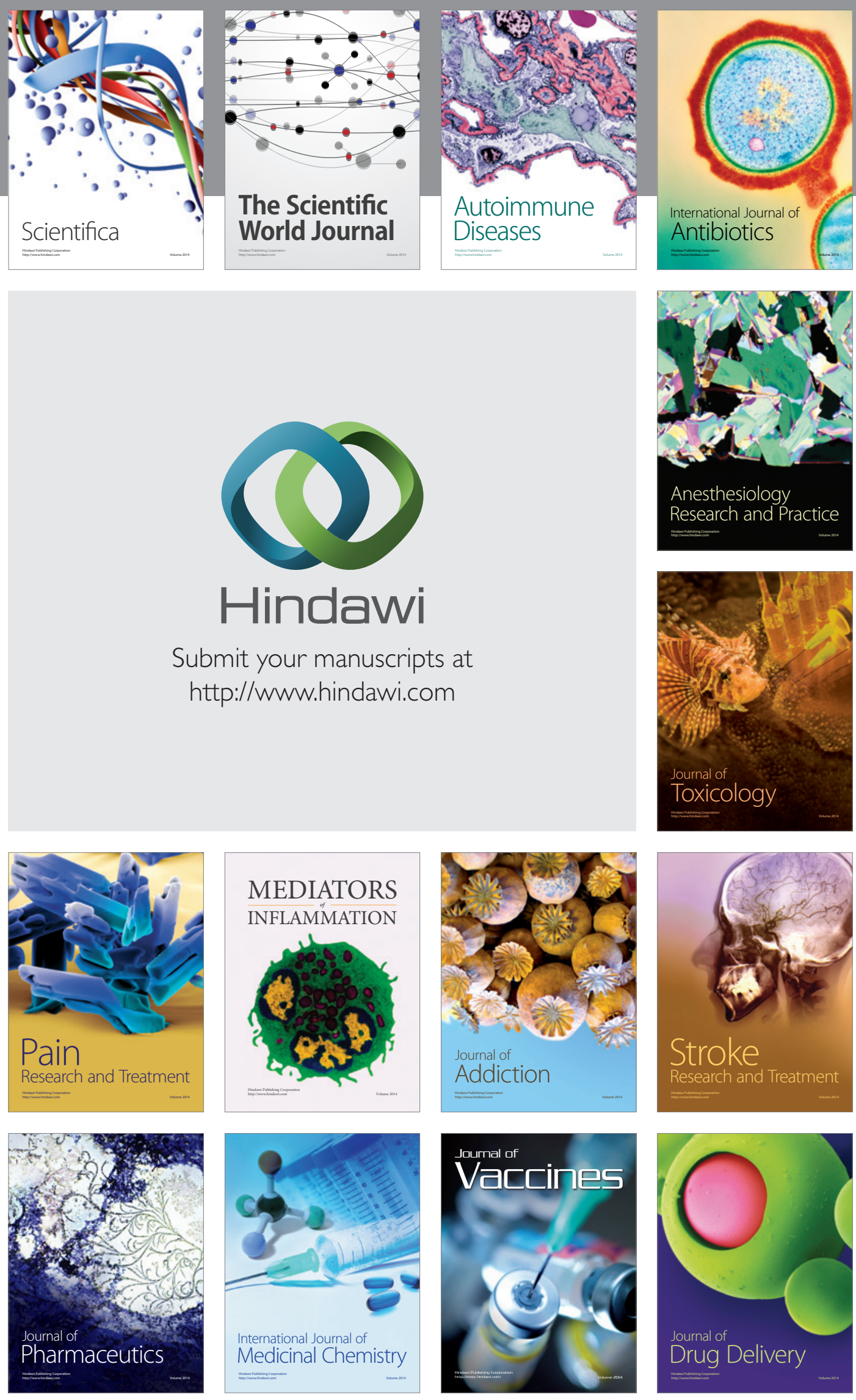